\title{
Noise Characteristics Test of Hy-Vo Silent Chain for Hybrid Vehicles
}

\author{
Yabing Cheng ${ }^{1, a^{*}}$, Xiaopeng Wang ${ }^{1, b}$, Honggang Qi ${ }^{2, c}$, Lei $\mathrm{Li}^{1, \mathrm{~d}}$, Zhenming Fu ${ }^{3, \mathrm{e}}$, Nen Wan ${ }^{1, \mathrm{f}}$ \\ ${ }^{1}$ College of Mechanical Science and Engineering, Jilin University, Changchun, China \\ ${ }^{2}$ China FAW Group Corporation Research and Design Center, China \\ ${ }^{3}$ Qingdao Zhenghe Industry Co., Ltd, China \\ a*chengyb@jlu.edu.cn, bwangxp2017jlu@163.com, ${ }^{\mathrm{c}} 1029854490 @ q q . c o m,{ }^{d} l e e l e i \_2014 @ 163 . c o m$, \\ e279364201@qq.com, ${ }^{\mathrm{f}} 952262495 @ q q . c o m$
}

Keywords: Hybrid vehicle; meshing theory; Hy-Vo silent chain; noise characteristics

\begin{abstract}
Based on the structure of hybrid vehicle and meshing theory of silent chain, the Hy-Vo silent chain drive system for hybrid vehicles is built. Through performing the noise characteristics test of Hy-Vo silent chain drive system, the noise value and noise characteristics under different rotational speeds are tested and researched. The test results showed that the noise value of $\mathrm{Hy}-\mathrm{Vo}_{\mathrm{O}}$ silent chain was in a normal level, which verified that the design of Hy-Vo silent chain system for hybrid vehicles is reasonable and feasible.
\end{abstract}

\section{Introduction}

Hybrid vehicles not only take advantage of the dynamic performance of internal combustion engine to maintain the high power output, but also possess the advantages of electromotor's low noise and non-pollution. Their dynamic characteristics, economic efficiency and environmental protection behavior could satisfy the requirements of new energy automobile raised by modern society.

As a new type of drive system, the Hy-Vo silent chain is widely used in hybrid vehicles. The noise characteristics of this drive technique reflect the reasonability of design and manufacturing technique of silent chain. There are some important research achievements about the design and noise characteristics of Hy-Vo silent chain. Meng Fanzhong, et al. systematically explained the meshing theory of silent chain, and studied the dynamic characteristics of roller chain and $\mathrm{Hy}-\mathrm{Vo}$ silent chain [1-7]. Troedsson I., et al. researched the vibration and loading of chain drive and established the model working at moderate and high speed conditions [8, 9]. Pereira C.M., et al. built an automatic multi-body model of chain drive from a minimal set of data, aiming at overcoming the difficulty on manually building the complex models of chain drives [10-12]. Masakazu wada, et al. mainly studied a single-stage cam drive system and improved the noise and vibration problems of chain system [13]. Masahiro Koizumi and Motoyasu Sakaguchi, et al. built the multi-body dynamic simulation model of timing silent chain transmission and increased the prediction accuracy of fluctuation and noise value in the process of transmission [14-15]. However, there are few reports about the research in noise experiment of Hy-Vo silent chain for hybrid vehicles. The Hy-Vo silent chain researched in this paper is used for power transmission between electromotor and gearbox for hybrid vehicle. Through performing the noise experiments, the noise value is tested and analyzed in real time under different rotational speeds, and the results could provide a guidance for the design analysis and noise experiment of other chain drive systems.

\section{Meshing design of Hy-Vo silent chain}

2.1 Parameters choose of Hy-Vo silent chain. The diagram of Hy-Vo silent chain is shown in Fig. 1. The chain plate pitch is $p=9.525 \mathrm{~mm}$; the plate pressure angle is $\alpha=30^{\circ}$; the datum apothem 
is $f=4.76 \mathrm{~mm}$; the circle center distance of chain plate hole is $A=8.188 \mathrm{~mm}$; the radius of large end surface of heterogeneous pin is $r=7.611 \mathrm{~mm}$; the distance from the center of plate hole to the large end surface of heterogeneous pin is $S_{m}=0.695 \mathrm{~mm}$; and the fixed angle of the heterogeneous pin is $\gamma=4.597^{\circ}$. The sprocket pitch is $p_{1}=9.525 \mathrm{~mm}$; and the sprocket pressure angle is $\alpha_{1}=31.5^{\circ}$.

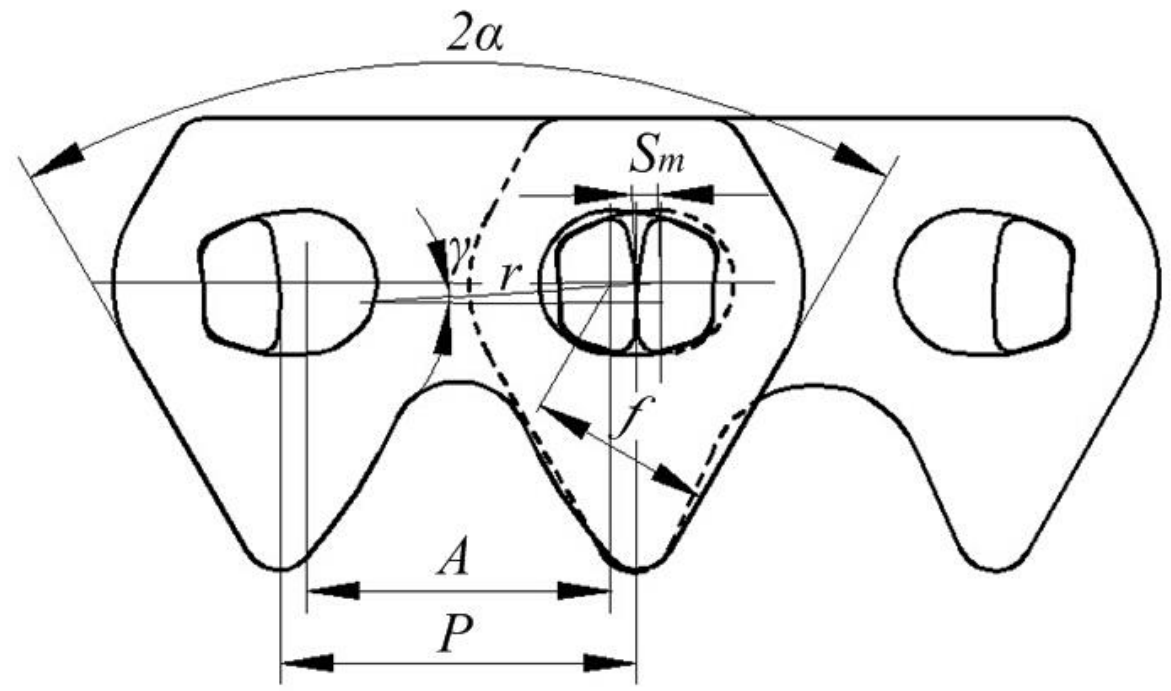

Figure 1. Diagram of Hy-Vo silent chain

2.2 Layout design of $\mathbf{H y}-\mathrm{Vo}$ silent chain drive system. According to the basic pitch $p=9.525 \mathrm{~mm}$, the thickness of chain plate $t=1.3 \mathrm{~mm}$, and the drive system space requirements of hybrid vehicles, the assembly space of chain is chosen as $7 \times 8$. The center distance of the sprockets is $a=242.4 \mathrm{~mm}$, the number of sprocket teeth of electromotor shaft $z_{1}=21$, and the number of shaft sprocket teeth of gearbox is $z_{2}=25$. The number of plates could be obtained by Eq. 1 .

$$
T=2 a_{o p}+\frac{z_{1}+z_{2}}{2}+\frac{c}{a_{o p}}
$$

where $a_{o p}=\frac{a}{p}, \quad c=\left(\frac{z_{2}-z_{1}}{2 \pi}\right)^{2}$.

After calculating the number of plates, we have $T=73.91$, and the number of plates is chosen $T_{0}=74$. According to the results, the schematic diagram of Hy-Vo silent chain drive system is shown in Fig. 2.

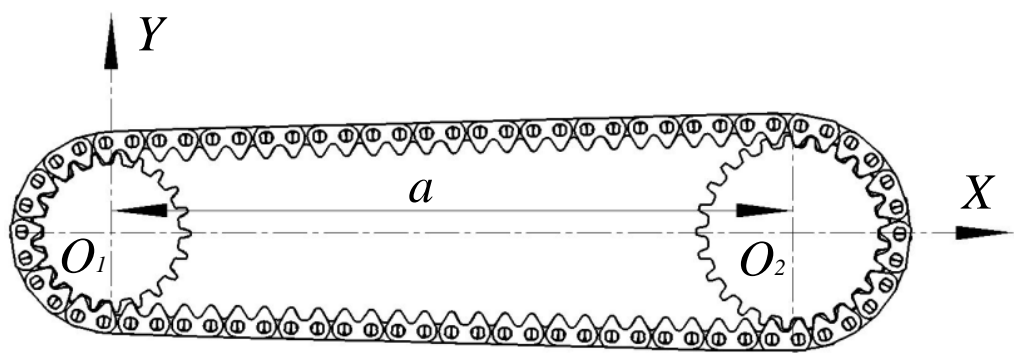

Figure 2. Schematic diagram of Hy-Vo silent chain drive system

\section{Noise test}

In recent years, automobile manufacturers have raised increasingly strict requirements on the overall noise index, which becomes an important criterion to evaluate a car's performance. Silent 
chain drive system is one of the main noise sources of car. Therefore, silent chain not only should have good transmission performance, sufficient strength and excellent abrasion resistance, but also could meet the requirements of noise level.

3.1 Test equipment and conditions. Noise test was completed in the noise test platform of chain drive system, as shown in Fig. 3. The test platform used the closed power flow theory and the rotational speed can reach $8000 \mathrm{r} / \mathrm{min}$. This high speed chain drive test platform can simulate the actual working status of silent chain to make noise test under variable speed and load. The measuring instrument used in the test is a portable two-channel noise and vibration spectrum analyzer AWA6290A, which can perform the real-time measurement of noise levels, as shown in Fig. 4.

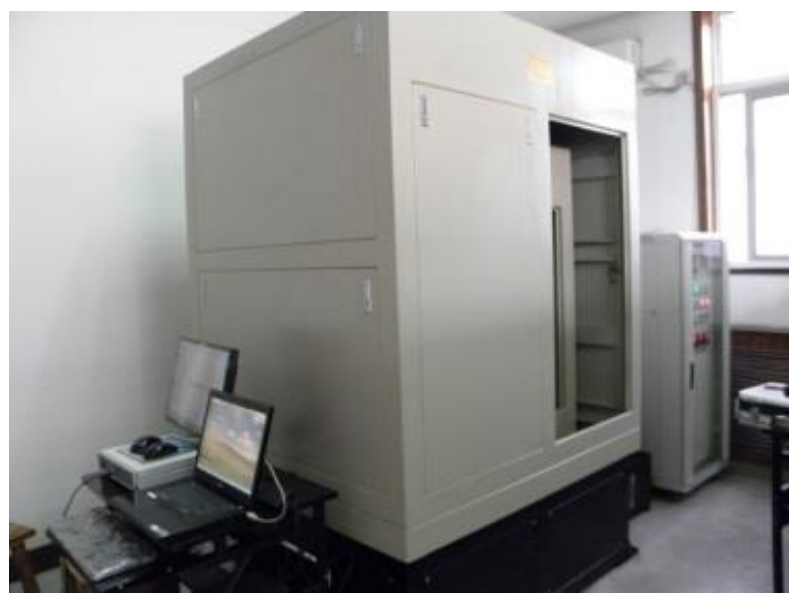

Figure3. Hy-Vo silent chain noise testing equipment

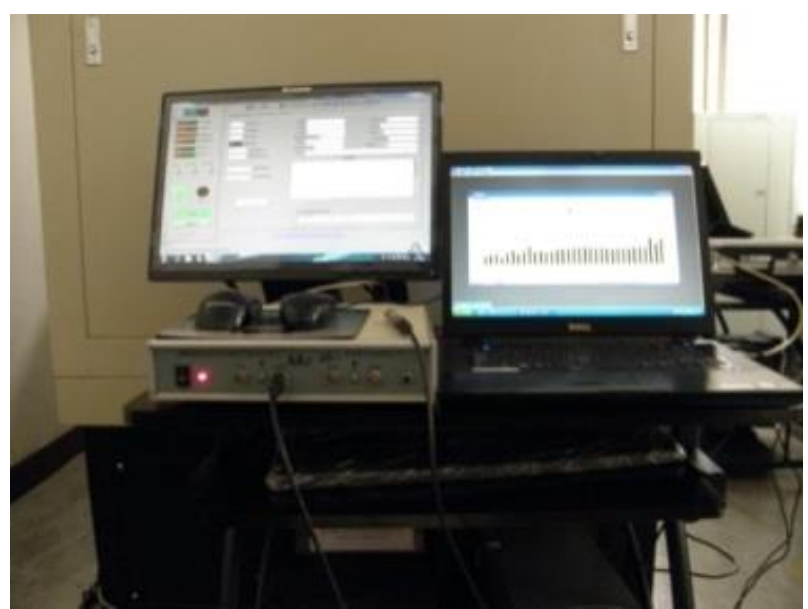

Figure 4. AWA6290A noise testing and analysis equipment

The test speed range of the drive sprocket is $1000 \sim 8000 \mathrm{r} / \mathrm{min}$, the noise is measured at an interval of $1000 \mathrm{r} / \mathrm{min}$, and the test load is $350 \mathrm{~N}$. The number of sprocket teeth is $z_{1}=21, z_{2}=25$; the type of the chain is $7 \times 8$ rows; and the number of chain links is 74 . The test assembly is shown in Fig.5. 


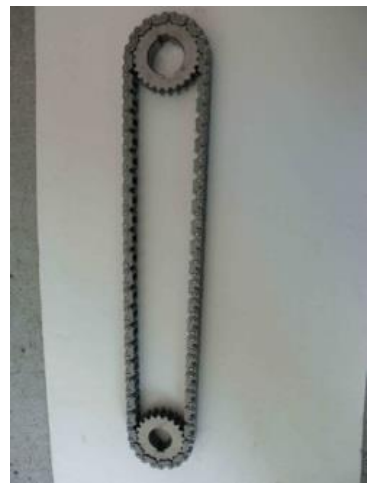

(a) Chain and sprockets

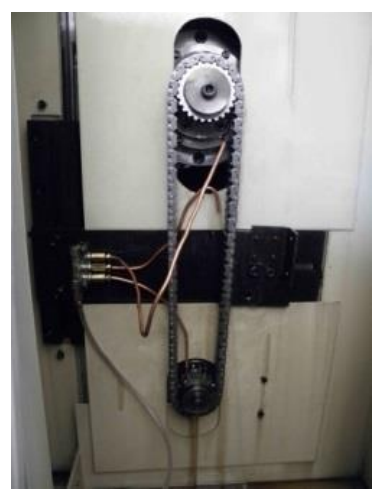

(b) Assembly of chain and sprockets

Figure 5. Tested chain and sprockets.

3.2 Measurement and analysis of test data results. The test environment usually has other interference noise called background noise. The chain noise needs to remove the background noise from the actually measured data. Usually the correction formula of background noise can be used to obtain the true noise level of noise source. Correction formula for background noise is shown in Eq.2.

$$
L_{a}=L_{P}-L_{Y}=L_{P}-10 \lg \left(1+\frac{1}{10^{\frac{L_{X}}{10}}-1}\right) .
$$

where $L_{a}$ - the real noise from the noise source

$L_{P}$ —composite noise

$L_{Y}$-modified value of background noise

$L_{X}$ - difference of composite noise and background noise

Under the same test conditions, the background noise value is equal to the noise value without chain and the composite noise value is the noise value when testing with chain. In this experiment, the measured value of noise is expressed by A weighted sound pressure level. Figure 6 is the curve graph of background noise at different rotational speeds and Figure 7 shows the composite noise values of Hy-Vo silent chain drive system.

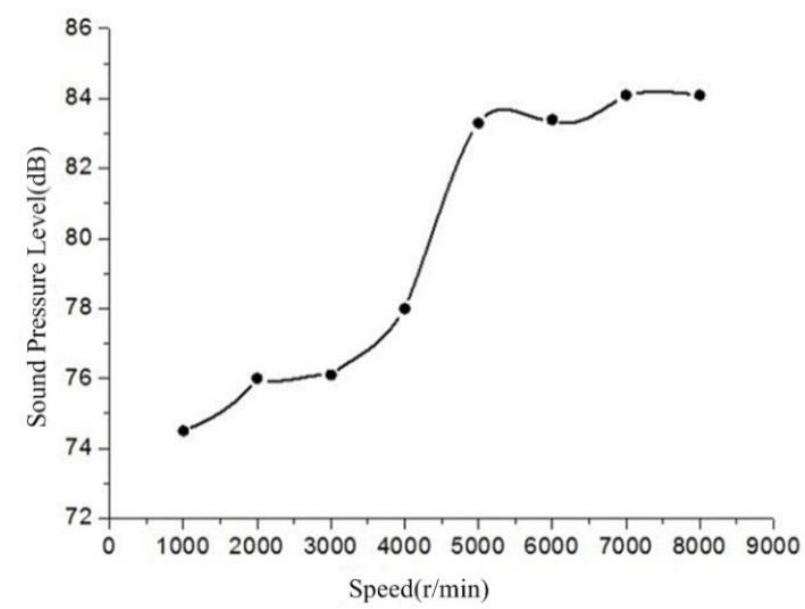

Figure 6. Background noise values under different speeds 


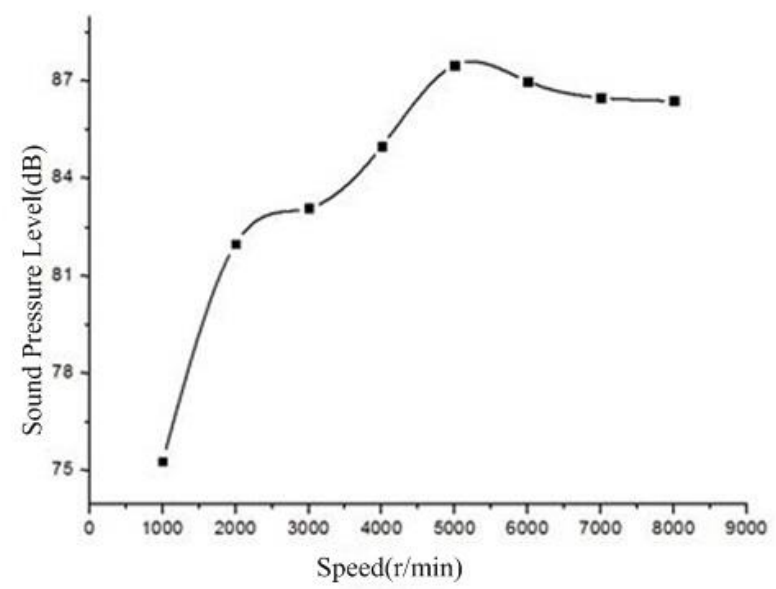

Figure 7. Composite noise values of system under different speeds

3.3 Experiment result analysis. Figure 8 is the noise value curve after removing the background noise. It can be seen that the largest noise value is about $84.8 \mathrm{~dB}$ while the acceptable noise value by the industry is not more than $85 \mathrm{~dB}$, so it can meet the design requirement. When the rotational speed is about $5000 \mathrm{r} / \mathrm{min}$, there is a demarcation point at the noise value curve. When the speed is lower than $5000 \mathrm{r} / \mathrm{min}$, the noise value is increased with the speed and when the speed is over $5000 \mathrm{r} / \mathrm{min}$, the noise value decreases with the speed, and finally is leveled off. The noise value was tested under the conditions that the testing equipment was treated with noise reduction measures and the background noise was treated with amendment, so the noise was composed of the static noise and the transient noise. Steady vibration produces periodic harmonic noise or peak noise, and the generation of peck noise is due to the existence of resonance phenomenon. As shown in Figure 8 , when the speed is equal to $2000 \mathrm{r} / \mathrm{min}$ or $5000 \mathrm{r} / \mathrm{min}$, there is an obvious fluctuation in the noise value curve, because the meshing impact of silent chain transmission system under these speeds is somewhat coupled with the natural frequency of the test bed structure, causing the mechanical resonance phenomenon and making the noise value change greatly.

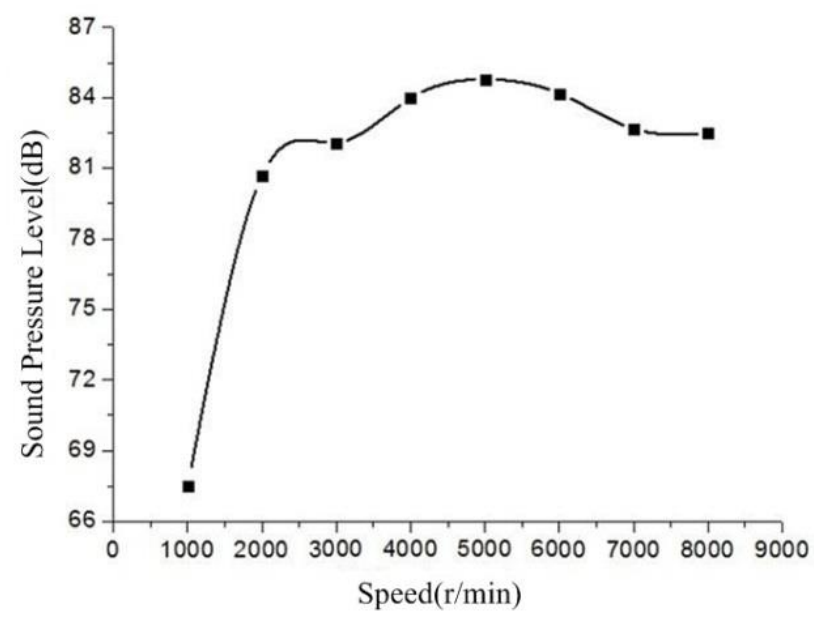

Figure 8. Noise values of Hy-Vo silent chain drive system for hybrid vehicles

Figure 9 is the noise frequency spectrum image of Hy-Vo silent chain drive system. From the testing results, the noise value under different frequencies demonstrates a periodical change because of the existence of steady vibration. When the center frequency is about $1600 \mathrm{H}_{\mathrm{Z}}$, the sound pressure level is largest and decreases with the increasing of frequency, which indicates that the vibration in this area is structural vibration and resonance phenomenon is serious. At this time, the noise of chain mainly comes from the noise stimulation when plates mesh with sprocket. Therefore, the working frequency of silent chain should be avoided in the structural resonance range in the process of silent chain transmission. The noise value in high frequency range is large while small at low 
frequencies. Therefore, to control the noise of transmission, the noise in high frequency range needs to be reduced.

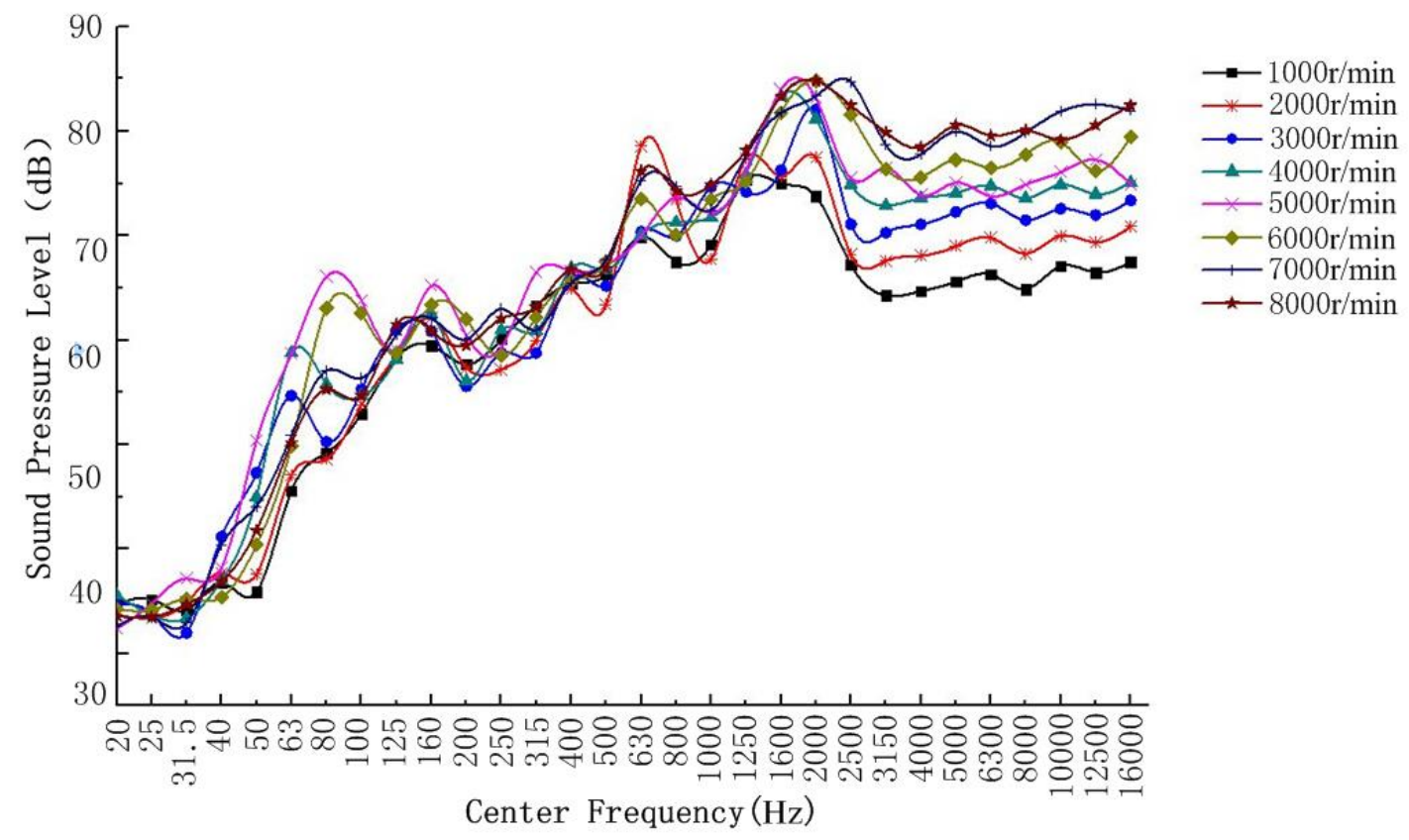

Figure 9. Noise frequency spectrum of Hy-Vo silent chain drive system for hybrid vehicles

\section{Conclusions}

(1) According to the drive system requirements of hybrid vehicles and the characteristics of Hy-Vo silent chain, the related parameters of Hy-Vo silent chain are chosen and the Hy-Vo silent chain drive system for hybrid vehicles is built.

(2) The noise value curve and noise frequency spectrum of Hy-Vo silent chain drive system are obtained by noise characteristic test of Hy-Vo silent chain drive system for hybrid vehicles. The analysis results show that the Hy-Vo silent chain drive system for hybrid vehicles can meet the noise requirement of industry.

(3) When the rotational speeds is equal to $2000 \mathrm{r} / \mathrm{min}$ or $5000 \mathrm{r} / \mathrm{min}$, there is an obvious fluctuation in the noise value curve, and there is resonance phenomenon. To shift the resonant frequency of system, the system structure or the connection of structural support should be improved. In addition, when the center frequency is equal to about $1600 \mathrm{~Hz}$, the sound pressure level is largest. It shows that this area is just in the structural resonance area, so the working frequency of Hy-Vo silent chain should be avoided in the structure resonance range.

\section{Acknowledgements}

The research presented in this paper was supported by National Natural Science Foundation of China (No. 51305154) and Science and Technology Development Project of Jilin Province in China (No.20116004, No. 20150204075GX).

\section{References}

[1] F.Z. Meng, Z.M. Feng and Y.X. Chu. 2006. Meshing theory and design method of new silent chain and sprocket, Chinese Journal of Mechanical Engineering, 19 (3): 425-427.

[2] F.Z. Meng, C. Li and Y.B. Cheng. 2007. Proper conditions of meshing for Hy-Vo silent chain and sprocket, Chinese Journal of Mechanical Engineering, 20 (4): 57-59. 
[3] F.Z. Meng, S.K. Wang, H. Lan, R. Xu and H.X. Xu. 2005. Wear failure mechanism and multi-impact property of automotive engine chain, Chinese Journal of Mechanical Engineering, 18:401- 404.

[4] F.Z. Meng, X. Liu and S. Xu. 2008. Meshing analysis and design of rotundity-datum-aperture Hy-Vo silent chain. Proceedings of the Institution of Mechanical Engineers, Journal of Mechanical Engineering Science, 222:1297-1303.

[5] Y.B. Cheng, F.Z. Meng, H.X Xu and J.M. Wu. 2007. Experimental study on high-speed characteristics of automotive engine, Chinese Journal of Mechanical Engineering, 20:36-39.

[6] Z.M. Feng, F.Z. Meng and C.T. Li. 2005.The meshing mechanism and simulation analysis of a new type silent chain, Journal of Shanghai Jiaotong University, 39 ( 9): 1427-1430.

[7] Q.H. LI, F.Z. Meng and Z.M. Feng. 2009. Research on noise test of new Hy-Vo silent chain, China Mechanical Engineering, 20:131-133.

[8] I. Troedsson and L. Vedmar. 2001. A dynamic analysis of the oscillations in a chain drive, ASME, Journal of Mechanical Design, 123 (3): 395-401.

[9] R. Stephenson, D. Glennie, J.N. Fawcett and J.M. Hale. 2000. A method of measuring the dynamic loads in high-speed timing chains, Journal of Automobile Engineering, 214 (2): 217-226. [10] C.M. Pereira, J.A. Ambrósio and A.L. Ramalho. 2010. A methodology for the generation of planar models for multibody chain drives, Multibody System Dynamics, 24 (3): 303-324.

[11] A.L. Schwab, J.P. Meijaard and P. Meijers. 2002. A comparison of revolute joint clearance models in the dynamic analysis of rigid and elastic mechanical systems, Mechanism and Machine Theory, 37 (9): 895-913.

[12] P. Flores, J. Ambrósio, J.C.P. Claro, H.M. Lankarani and C.S. Koshy. 2009. Lubricated revolute joints in rigid multibody systems, Nonlinear Dynamics, 56 (3): 277-295.

[13] W. Masakazu, I. Shoichiro, M. Sadao and E. Atsushi. 1999. Development of a Small Pitch Silent Chain for a Single-Stage Cam Drive System, SAE Technical Paper Series, 1:1-7.

[14] K. Masahiro, M. Yuma and K. Makio. 2005. Muti-body Dynamics Simulation of Valve Train and Timing Chain Drive, Automotive Engineering, 135: 7-10.

[15] S. Motoyasu, A. Masaki, Y. Shinji, et al. 2010. Study of Chain Meshing Noise Using Multi-body Dynamics Simulation, Automotive Engineering, 116: 17-20. 